\title{
27. RADIAL VELOCITIES OF NEUTRAL HYDROGEN IN THE ANTICENTER REGION OF THE GALAXY
}

\author{
L. VELDEN
}

Astronomische Institute der Universität Bonn, Bonn, Germany

\begin{abstract}
An observational material of 21-cm Hı emission-line profiles is investigated by a statistical method to derive the kinematical properties of the interstellar gas in the region of the galactic anticenter. A description of the method used as well as the results obtained, concerning deviations from a circular rotation, are given.
\end{abstract}

This report on radial velocities of the neutral hydrogen in the anticenter region is based on 1253 observations of $21 \mathrm{~cm}$-emission-line profiles. The observations were done at the 25-m telescope of the University of Bonn (Velden, 1970).

Figure 1 shows a schematic diagram of the observational program in galactic coordinates. The observations are arranged as 11 cross sections of the galactic plane, indicated by vertical lines in the longitude range $l^{\mathrm{II}}=120^{\circ}$ to $240^{\circ}$ with a spacing of $10^{\circ}$. Each cross section consists of line profiles observed at fixed positions in the latitude range from $-30^{\circ}$ to $30^{\circ}$ with a spacing of $\frac{1}{2}^{\circ}$ in latitude. No observations were done at longitudes $160^{\circ}$ and $210^{\circ}$. The dashed horizontal lines separate the observed positions into three latitude ranges later used.

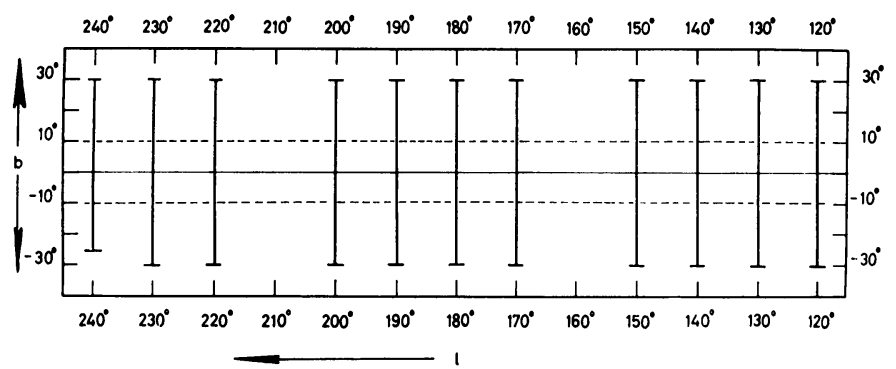

Fig. 1. Schematic diagram in galactic coordinates of the region observed. The vertical lines indicate the cross sections covered by fixed position $21-\mathrm{cm}$ observations.

The observations were done to win a broad base for a statistical investigation of the neutral hydrogen properties as seen under the influence of varying differential galactic rotation. A further intention was to derive criteria for the known spiral features outside the anticenter and try to follow them through the anticenter region. The investigations done for this purpose showed, though not quite unexpectedly, a kinematical effect, the description of which was made the object of this paper.

The reduction procedure (Schwartz, 1967; Grahl et al., 1968) yielded the line profiles in the form of brightness temperature vs. radial velocity with respect to the local standard of rest. For the velocity reduction the standard solar motion was used. 
The kinematical investigation was done in a strictly statistical sense. The line profiles of a latitude range at a fixed longitude were used for the calculation of a mean velocity. This was achieved by weighting the radial velocities of the line profiles by their respective brightness temperatures. For a latitude range the mean velocity thus derived may be called 'profile area weighted mean velocity'. To smooth out the random motions of individual objects and derive the large scale motions mean radial velocities were determined for latitude ranges of at least $20^{\circ}$.

The method of area-weighting used is handicapped against calculating mass-weighted mean velocities, by giving less weight to the same physical objects at greater distances. On the other hand the calculation of mass-weighted mean velocities would be based on, especially in the anticenter region, highly doubtful individual distances derived from a model of galactic rotation.

An advantage of the method used is the inclusion of the material at velocities forbidden in the sense of a circular rotational model.

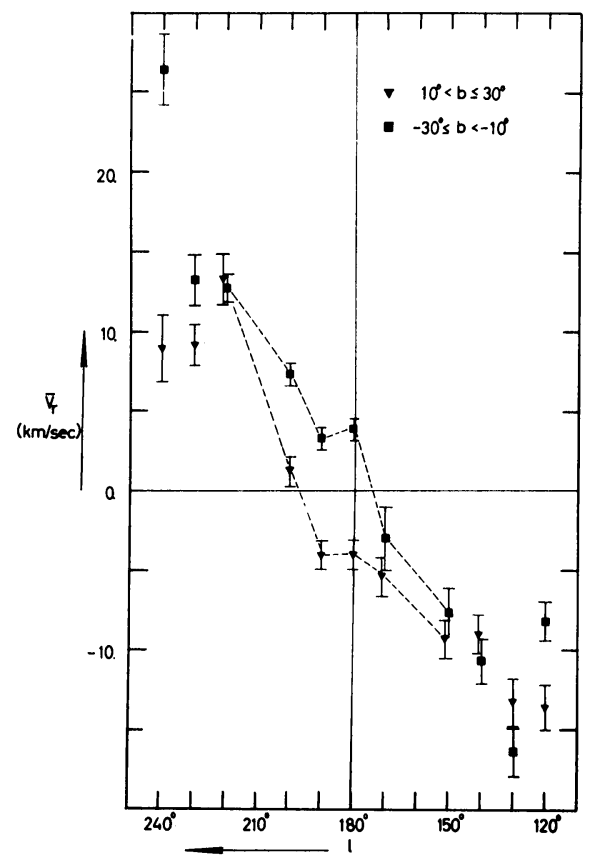

Fig. 2. Velocity-longitude diagram of the observations at intermediate latitudes above $\left(10<b^{\mathrm{II}} \leqslant 30\right.$, triangles) and below $\left(-30 \leqslant b^{\mathrm{II}}<-10\right.$, squares) the galactic plane. The error bars indicate the computed rms-errors.

Figure 2 shows a velocity-longitude diagram for the mean velocity points of the latitude ranges $-30^{\circ}$ to $-10^{\circ}$ represented by squares and $10^{\circ}$ to $30^{\circ}$ plotted as triangles. The error bars indicate the computed rms errors. The velocities above and below the galactic plane show a splitting around the anticenter of $\pm 4.5 \mathrm{~km} \mathrm{~s}^{-1}$. The extension of the velocity splitting to higher and lower galactic longitudes remains 
uncertain since the line of sight does there obviously cut into more distant material because of the known bending of the galactic plane. The velocity splitting at the anticenter can be removed by a correction for a relative velocity of gas and LSR of $-12.5 \mathrm{~km} \mathrm{~s}^{-1}$ in the direction of the galactic north pole.

Figure 3 demonstrates the mean velocity-longitude relation of the same intermediate latitude observations combined now into one class as open circles. A formal correction of $12.5 \mathrm{~km} \mathrm{~s}^{-1}$ of the LSR velocity in the galactic pole direction was applied. A rough fit to the mean velocity points is achieved by the dashed curve which was calculated from Schmidt's model of galactic rotation for material assumed at a distance of $0.8 \mathrm{kpc}$ from the sun in the galactic plane.

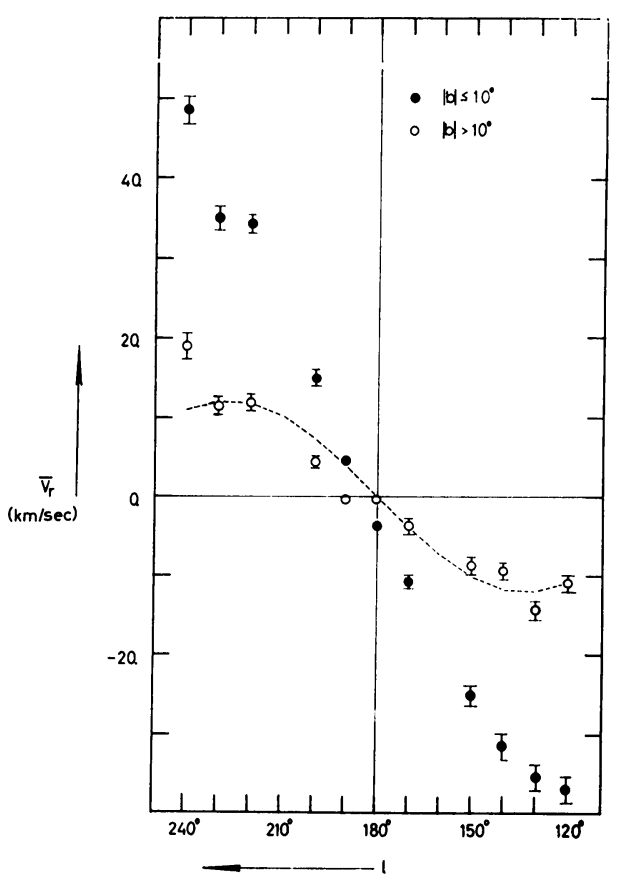

Fig. 3. Velocity-longitude diagram of the combined intermediate latitude observations $\left(10<\left|b^{\mathrm{II}}\right| \leqslant 30\right.$, open circles) and the plane observations $\left(0 \leqslant 10\left|b^{\mathrm{II}}\right| \leqslant 10\right.$, filled circles).

The filled circles represent the mean velocities of the low latitude observations between $-10^{\circ}$ and $10^{\circ}$. Though a rather smooth curve can be drawn for their velocitylongitude relation, a systematic deviation to negative velocities of about $3.5 \mathrm{~km} \mathrm{~s}^{-1}$ is apparent near the anticenter.

Figure 4 shows the influence of this on a distance determination in a polar diagram of distance from the sun vs. galactic longitude. The mean velocities of the low latitude class are transformed to mean distances by Schmidt's model of circular galactic rotation and plotted as open circles. A significant distance jump does appear at the anticenter direction. Recalculating the mean distances, under the idea of an 


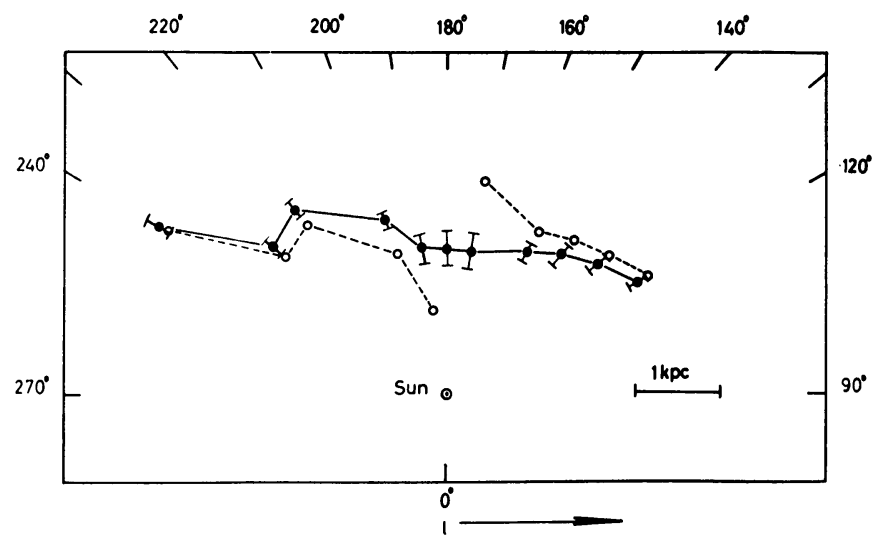

Fig. 4. Polar diagram of distance from the sun vs. galactic longitude. The open circles represent the mean velocity points of Figure 3 of the plane material transformed by Schmidt's (1965) rotational model to mean distance points. The filled circles show the mean distances after a formal correction for an outward motion of the LSR of $3.3 \mathrm{~km} \mathrm{~s}^{-1}$.

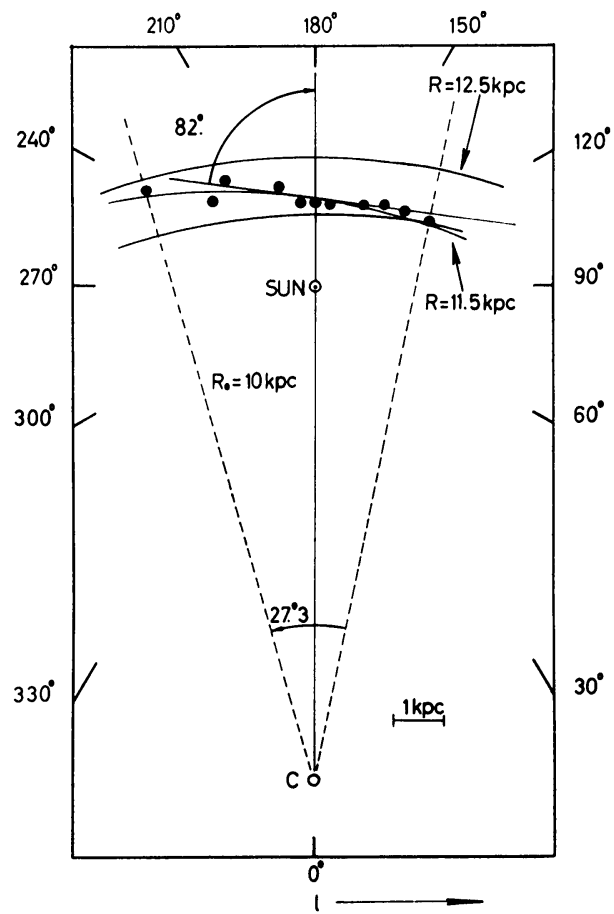

Fig. 5. Polar diagram of mean distances from the galactic center vs. longitude of the plane observations. 
in general smooth spatial distribution of the $\mathrm{H}_{\mathrm{I}}$ gas, for an assumed outward motion of the LSR of $3.3 \mathrm{~km} \mathrm{~s}^{-1}$ yields the line of filled circles. The distance for the anticenter point is derived from the slope of the mean velocity curve.

Figure 5 does need a warning because of its rather suggestive character. The mean distance points are plotted with respect to the galactic center. An increase of mean distances of $0.8 \mathrm{kpc}$ is found for a range of $27^{\circ}$ of galactocentric longitude. A calculated mean distance line for the points is inclined to the center-anticenter line with $82^{\circ}$.

The observational facts presented are summed up by the following.

(1) The mean radial velocities observed at intermediate galactic latitudes are roughly described by a circular rotation law. The splitting of the velocity-longitude curves of the latitude ranges above and below the plane indicates a relative velocity of $12.5 \mathrm{~km} \mathrm{~s}^{-1}$ in $z$-direction between $\mathrm{HI}_{\mathrm{I}}$ gas and assumed LSR near the anticenter.

(2) A motion of approach of $3.3 \mathrm{~km} \mathrm{~s}^{-1}$ between Hi gas of low latitude and LSR is observed in the anticenter region, which does not seem present in the gas at intermediate latitudes.

(3) The mean distance of the plane material increases in general with increasing galactic longitude in the region observed.

(4) The slight bulge of the mean distance points towards lower distances from the sun near the anticenter direction indicates an only low value for the self-absorption of the $21-\mathrm{cm}$ emission line.

\section{References}

Grahl, B. H., Hachenberg, O., and Mebold, U.: 1968, Beiträge zur Radioastronomie 1, 3.

Schmidt, M.: 1965, Stars and Stellar Systems 5, 513.

Schwartz, R.: 1967, Forschungsberichte des Landes Nordrhein-Westfalen, Nr. 1844, Westdeutscher Verlag Köln-Opladen.

Velden, L.: 1970, 'Atlas of 21-cm Line Profiles of the Outer Part of the Galaxy in the Region of the Galactic Anticenter', Beiträge zur Radioastronomie, in preparation. 